\section{7 \\ WORKPLACE VIOLENCE AGAINST NURSES IN HOSPITALS IN GUANGDONG, CHINA}

doi:10.1136/injuryprev-2012-040590m.7

ZH Chen, HY Xia. First Affiliated Hospital of Jinan University, 613 West Huangpu Avenue, Guangzhou, China

Background Violent incidents can appear everywhere including hospitals. Nurses are the main working population in health facilities with female gender mostly. How is workplace violence against this special group in Guangdong Province?

Objective To examine the epidemiological features of nurse workplace violence.

Methods A retrospective analysis was carried out on hospital nurses from four different cities in Guangdong Province.

Results Among 3319 nurses investigated, 2085 have been subjected to workplace violence in the past 12 months preceding this survey with a rate of $62.82 \%$, accounting for $62.07 \%$ of psychological violence and $15.06 \%$ of physical violence respectively. Most workplace violence incidents happened in Accident and Emergency departments (78.71\%). Nurse stations (53.61\%) and wards (28.62\%) were two leading places of workplace violence. One third of workplace violence incidents came forth during night shift. Male nurses were more vulnerable to workplace violence against female ( $\chi^{2} 3.263$; $p$ $0.017)$. Those victims below 40 years were at greatest risk of violence (OR 3.274; 95\% CI 2.417 to 3.871).

Significance Magnitude of nurse workplace violence in hospitals in Guangdong Province and necessity of taking comprehensive measures with nurse individuals, hospitals, government and the Media working together for workplace violence prevention and control. 